\title{
An Efficient Cluster-Based Power Saving Scheme for Wireless Sensor Networks
}

\author{
Kasa Suguna \\ M.Tech in Computer Science and Engineering from JNTU Hyderabad and Pursuing Ph.d in Rayalaseema University, Kurnool, A.P, India
}

\begin{abstract}
In this article, efficient power saving scheme and corresponding algorithm must be developed and designed in order to provide reasonable energy consumption and to improve the network lifetime for wireless sensor network systems. The clusterbased technique is one of the approaches to reduce energy consumption in wireless sensor networks. In this article, we propose a saving energy clustering algorithm to provide efficient energy consumption in such networks. The main idea of this article is to reduce data transmission distance of sensor nodes in wireless sensor networks by using the uniform cluster concepts. In order to make an ideal distribution for sensor node clusters, we calculate the average distance between the sensor nodes and take into account the residual energy for selecting the appropriate cluster head nodes. The lifetime of wireless sensor networks is extended by using the uniform cluster location and balancing the network loading among the clusters. Simulation results indicate the superior performance of our proposed algorithm to strike the appropriate performance in the energy consumption and network lifetime for the wireless sensor networks.
\end{abstract}

Keywords: cluster, distance, power consumption, lifetime, sensor networks

\section{Introduction}

Recently, there has been a rapid growth in the wireless communication technique. Inexpensive and low-power wireless micro-sensors are designed and widely used in wireless and mobile environments [1-3]._A wireless sen- sor network consists of a large number of sensor nodes. Each sensor node has sensing, computing, and wireless communication capability. All sensor nodes play the role of an event detector and the data router. Sensor nodes are deployed in the sensing area to monitor specific targets and collect data. Then, the sensor nodes send the data to sink or base station (BS) by using the wire- less transmission technique. Wireless sensor networks have been pervasive in various applications including health care system, battlefield surveillance system, envir- onment monitoring system, and so on. Figure 1 shows an infrastructure of wireless sensor networks. Power saving is one of the most important features for the sensor nodes to extend their lifetime in wireless sensor net- works. A sensor node consumes mostly its energy in transmitting and receiving packets. In wireless sensor networks, the main power supply of the sensor node is battery. However, in most application scenarios, users are usually difficult to reach the location of sensor nodes. Due to a large number of sensor nodes, the replacement of batteries might be impossible. However, the battery energy is finite in a sensor node and a sensor node draining of its battery may make sensing area uncovered. Hence, the energy conservation becomes a critical concern in wireless sensor networks. In order to increase energy efficiency and extend the network life- time, new and efficient power saving algorithms must be developed [4-6].

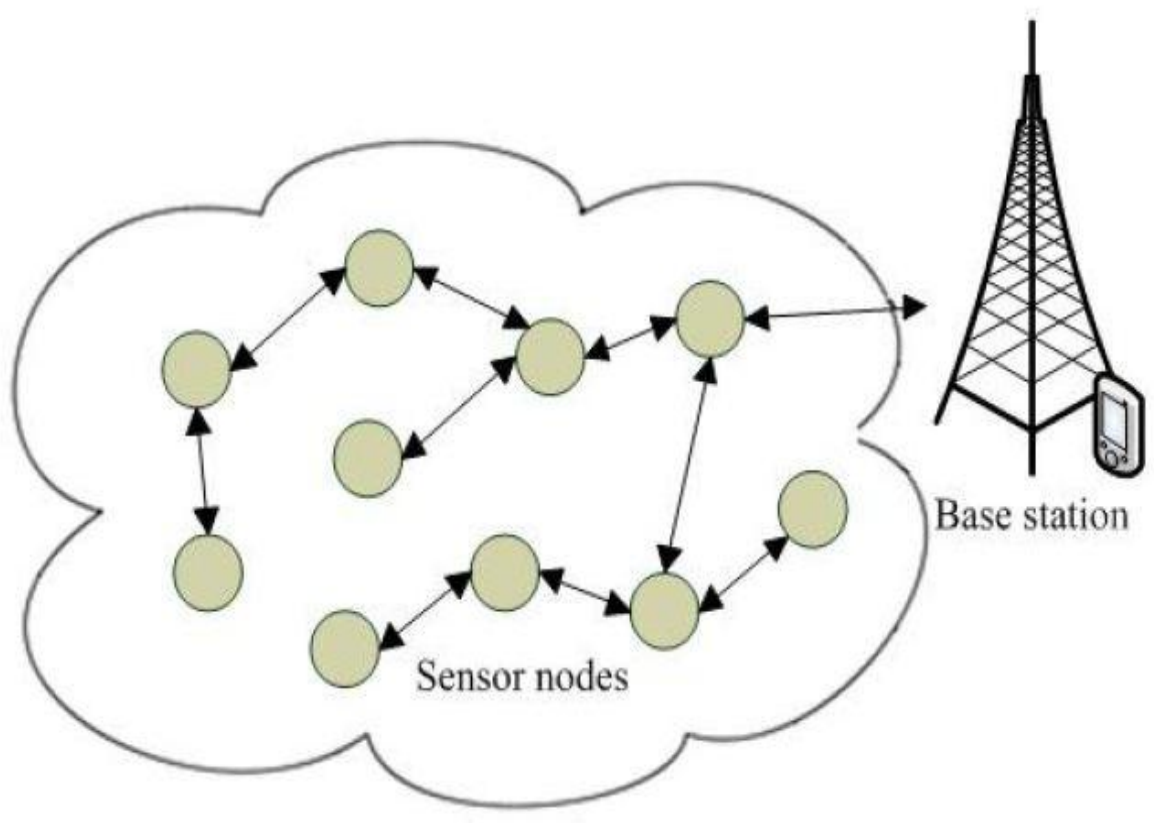

Figure 1 Infrastructure of wireless sensor networks. 


\section{International Journal of Science and Research (IJSR) \\ ISSN (Online): 2319-7064}

Index Copernicus Value (2013): 6.14 | Impact Factor (2014): 5.611

Low Energy Adaptive Clustering Hierarchy (LEACH) is a typical cluster-based protocol using a distributed clustering formation algorithm [5]. In LEACH, the large number of sensor nodes will be divided into several clusters. For each cluster, a sensor node is selected as a cluster head. The selection of cluster head nodes is based on a predetermined probability. Other non-cluster head nodes choose the nearest cluster to join by receiving the strength of the advertisement message from the cluster head nodes. A non-cluster head node can only monitor the environment and send data to its cluster head node. The cluster head node is responsible for collecting the information of non-cluster head nodes in the cluster. Then, it processes data and sends data to the BS. As a non-cluster head node cannot send data directly to the BS, the data transmission distance of the sensor node is shrunk. Therefore, the energy consumption is reduced in the wireless sensor networks. How- ever, the random selection of the cluster head node may obtain a poor clustering setup, and cluster head nodes may be redundant for some rounds of operation. The distribution of cluster head nodes is not uniform, thus some sensor nodes have to transfer data through a longer distance and the reasonable energy saving is not obtained in wireless sensor networks. LEACH-centralized (LEACH-C) is proposed as an improvement of LEACH which uses a centralized clustering algorithm to create the clusters [6].In LEACH-C, the BS collects the information of the position and energy level from all sensor nodes in the networks. Based on this information, the BS calculates the number of cluster head nodes and configures the network into clusters. In [7], the authors propose the hybrid; energy-efficient, distributed (HEED) clustering protocol to prolong the network life- time and support scalable data aggregation. In this protocol, the cluster heads are probabilistically selected based on their residual energy and the sensor nodes join the clusters according to their power level. The cluster- based power saving methods has been proposed in [8-11], which have been validated to some extent through simulation. However, the critical problem is that the cluster size is also not uniform in these schemes.
The energy consumption cannot improve effectively. Figures 2 and 3 show the extreme cases of Clustering structure for a certain round in the simulation by using LEACH.

In this article, we propose a saving energy clustering algorithm (SECA) to provide efficient energy consumption in wireless sensor networks. In order to make an ideal distribution for sensor node clusters, we calculate the average distance between the sensor nodes and take into account the residual energy for selecting the appropriate cluster head nodes. The lifetime of wireless sensor networks is extended by using the uniform cluster location and balancing the network loading among the clus- ters. The main benefits of proposed scheme are that the energy consumption is reduced and better network life- time can be carried out.

The rest of this article is organized as follow. In Section 2, we present the system model of wireless sensor networks. In Section 3, we illustrate the proposed scheme in detail. In Section 4, we present our simulation model and analyze the comparative evaluation results of the proposed scheme through simulations. Finally, some conclusions are given in Section 5 .

\section{System Model}

The system infrastructure is composed of a BS and some sensor nodes. We classify all sensor nodes into noncluster head nodes and cluster head nodes. The noncluster head nodes operate in sensing mode to monitor the environment information and transmit data to the cluster head node. Also, the sensor node becomes a cluster head to gather data, compresses it and for- wards to the BS in cluster head mode. The system framework of this article is shown in Figure 1.

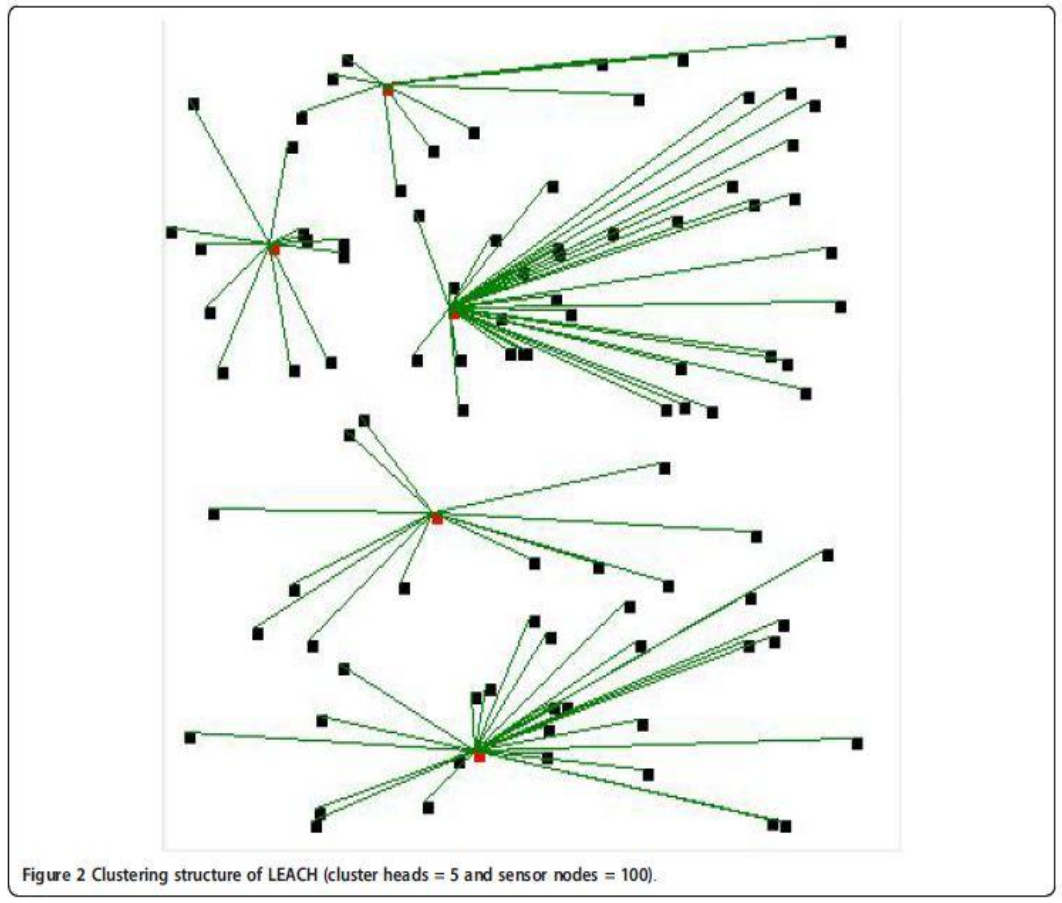

Volume 4 Issue 11, November 2015 


\section{International Journal of Science and Research (IJSR) \\ ISSN (Online): 2319-7064}

Index Copernicus Value (2013): 6.14 | Impact Factor (2014): 5.611

In wireless sensor networks, data communications consume a large amount of energy. The total energy consumption consists of the average energy dissipated by data transmission of the non cluster head nodes and the cluster head nodes. In addition, the energy consumption for data collection and aggregation of cluster head nodes is considered. Figure 4 illustrates the radio energy dissipation model in wireless sensor networks [5-7]. In this model, to exchange an L-bit message between the two sensor nodes, the energy consumption can be calculated by

$$
E_{T x}(L, d)=E_{\text {elec }} \times L+\varepsilon_{a m p} \times L,
$$

$$
E_{R x}(L)=E_{\text {elec }} \times L,
$$

where $\mathrm{d}$ is the distance between the two sensor nodes, $\operatorname{ETx}(\mathrm{L}, \mathrm{d})$ is the transmitter energy consumption, and $\operatorname{ERx}(\mathrm{L})$ is the receiver energy consumption. Eelec is the electronics energy consumption per bit in the transmit- ter and receiver sensor nodes. amp is the amplifier energy consumption in transmitter sensor nodes, which can be calculated by

$$
\varepsilon_{a m p}=\left\{\begin{array}{c}
\varepsilon_{f s} * d^{2}, \text { when } d \leq d_{0} \\
\varepsilon_{m p} * d^{4}, \text { when } d>d_{0}
\end{array},\right.
$$

where $\mathrm{d} 0$ is a threshold value. If the distance $\mathrm{d}$ is less than $\mathrm{d} 0$, the free-space propagation model is used. Otherwise, the multipath fading channel model is used. Efs and $\varepsilon \mathrm{mp}$ are communication energy parameters. Using the previously described in the literature [5,6], the $\varepsilon \mathrm{fs}$ is set as 10 $\mathrm{pJ} / \mathrm{bit} / \mathrm{m} 2$ and $\varepsilon \mathrm{mp}$ is set as $0.0013 \mathrm{pJ} / \mathrm{bit} / \mathrm{m} 4$. Also, the energy for data aggregation of a cluster head node is set as $\mathrm{EDA}=5 \mathrm{~nJ} / \mathrm{bit} / \mathrm{signal}$ and the initial energy of a sensor node is set as Einit $=2 \mathrm{~J}$. Suppose that a non-cluster head node $\mathrm{N}$ transmits $\mathrm{LN}$ bits to the BS. Let $\mathrm{dN}, \mathrm{CH}$ be the distance between the non-cluster head node $\mathrm{N}$ and its cluster head node $\mathrm{CH}$. Let $\mathrm{dCH}$, BS be the distance between the cluster head node $\mathrm{CH}$ and the $\mathrm{BS}$.

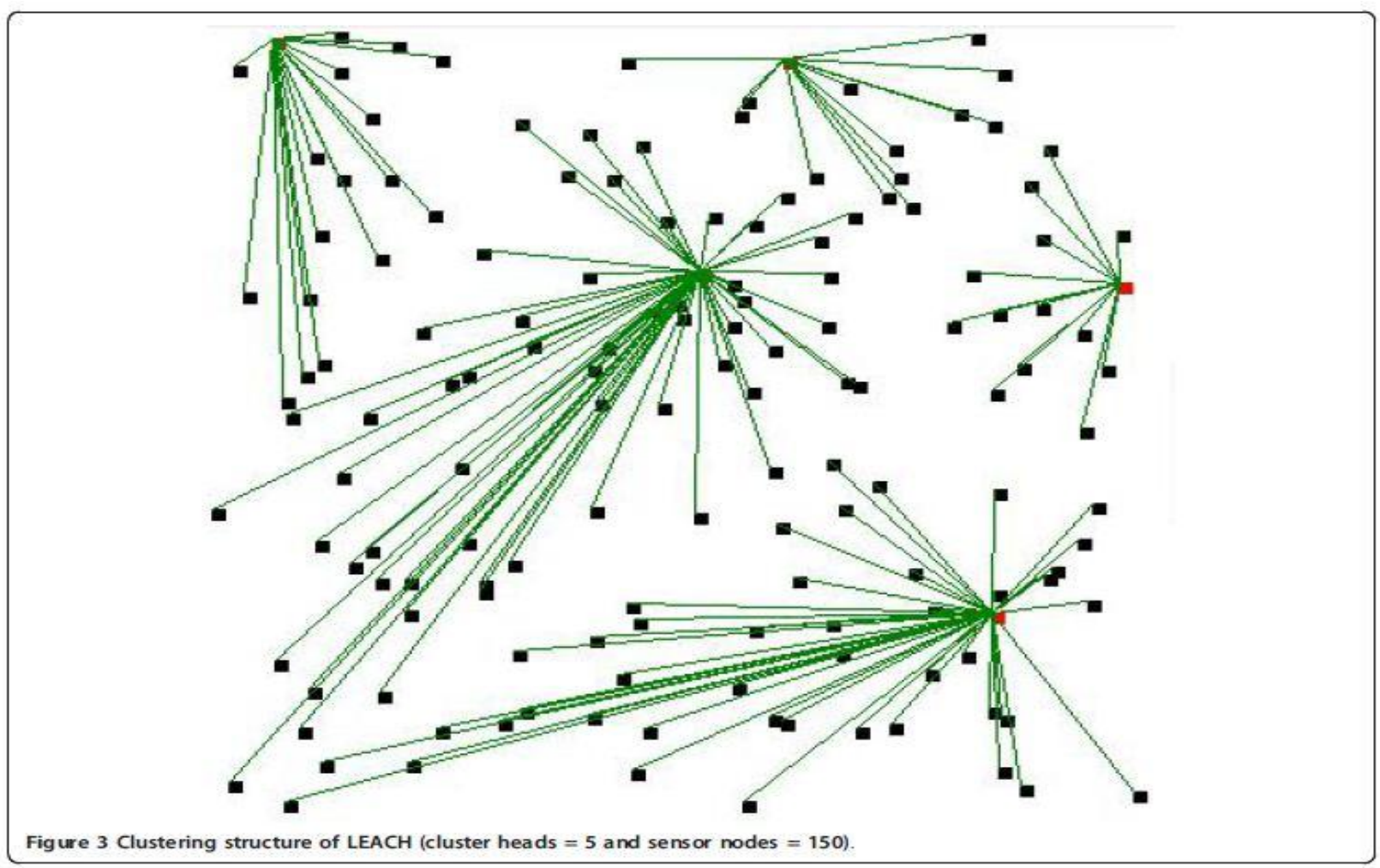

Due to the multi hop communication, a non- cluster head node only sends data to its cluster head node. The residual energy of the non-cluster head node $\mathrm{N}$ is equal to Einit $\operatorname{ETx}(\mathrm{LN}, \mathrm{dN}, \mathrm{CH})$. In addition, the resi- dual energy of the cluster head node $\mathrm{CH}$ is equal to Einit - ERx(LN) - EDA $\operatorname{ETx}(\mathrm{LN}, \mathrm{dCH}, \mathrm{BS})$, because the cluster head node must collect and process the information of non-cluster head nodes in the cluster, and then send data to the BS

It is obvious that the data transmission between sensor nodes takes most of the energy consumption in the wireless sensor networks. Taking into account the energy consumption of sensor nodes, the data transmission distance must be reduced and the packets delay should be avoided. Hence, the energy consumption and routing design become an important issue in the wire- less sensor networks.

\section{Proposed methods: SECA}

In order to increase energy efficiency and extend the lifetime of the sensor nodes in wireless sensor networks, efficient power saving algorithm must be developed and designed. Based on the centralized clustering architec- ture, we propose a SECA to provide efficient energy consumption and better network lifetime in the wireless sensor networks. In the proposed scheme, we assume that the BS receives the information of location and residual energy for each sensor node and the average residual energy can be calculated. When the residual energy of sensor node is higher than the

\section{Volume 4 Issue 11, November 2015}




\section{International Journal of Science and Research (IJSR) \\ ISSN (Online): 2319-7064}

Index Copernicus Value (2013): 6.14 | Impact Factor (2014): 5.611

average residual energy, the sensor node becomes a candidate of cluster head. We modify k-means algorithm to make an ideal distribution for sensor node clusters by using the information of location and residual energy for all sensor nodes $[12,13]$. In this algorithm, the operation includes two phases: set-up and steady-state phases.

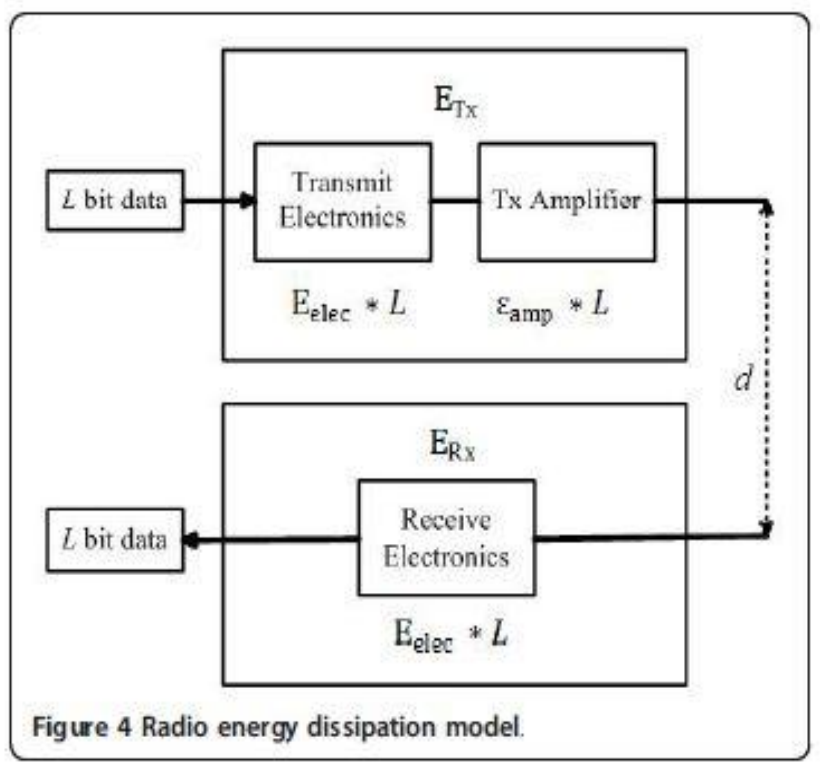

\subsection{Set-up phase}

The main goal of this phase is to create clusters and find cluster head nodes. During the set-up phase, the BS collects the information of the position and energy level from all sensor nodes in the networks. Based on the characteristics of stationary sensor nodes, the suitable initial means of points for clusters can be obtained. Let $\mathrm{C}$ be the center location for all sensor nodes. If there are $\mathrm{n}$ sensor nodes in the wireless sensor networks, $\mathrm{C}$ can be calculated by

$$
C=\frac{\sum_{i=1}^{n} X_{i}}{n},
$$

where $\mathrm{Xi}$ is the coordinate of sensor node $\mathrm{i}$.

Let $\mathrm{R}$ be the average distance between $\mathrm{C}$ and all sensor nodes, which can be calculated by

$$
R=\frac{\sum_{i=1}^{n}\left|X_{i}-C\right|}{n} .
$$

According to $\mathrm{C}$ and $\mathrm{R}$, the locations of initial mean of point mi(mix , miy $)$ for the cluster $i$ is calculated by

$$
\left\{\begin{array}{l}
m_{i_{x}}=R \times \cos \left(\frac{360}{k_{k}} \times(i-1) \times \frac{\pi}{180}\right)+C_{x} \\
m_{i_{y}}=R \times \sin \left(\frac{360}{k} \times(i-1) \times \frac{\pi}{180}\right)+C_{y}
\end{array},\right.
$$

where $\mathrm{k}$ is the number of clusters and $\mathrm{i}=1,2, \ldots, \mathrm{k}$. Figure5 shows the example of the initial means of points, where $\mathrm{k}$ is equal to 3 . The initial value $\mathrm{k}$ must be decided in the initial set-up phase. According to the definition of optimum number of clusters in LEACH-C [6], $\mathrm{k}$ can be calculated by

$$
k=\left\lceil\frac{\sqrt{n}}{\sqrt{2 \pi}} \sqrt{\frac{\varepsilon_{f s}}{\varepsilon_{m p}}} \frac{M}{d^{2} \text { to } B S}\right\rceil,
$$

Where $\mathrm{M}$ is the side of the given square field. The dto BSis the average distance from the cluster head nodes to the BS which is defined in LEACH-C. However, the cluster head nodes are selected by creating some clusters in our proposed algorithm. Hence, we re-define dto BS which is the average distance from the all sensor nodes to the BS.

The setting of initial means of points is very important. It can reduce the iteration time for creating clusters significantly. After the initial means of points are set, based on the location of all sensor nodes, the BS creates some clusters.

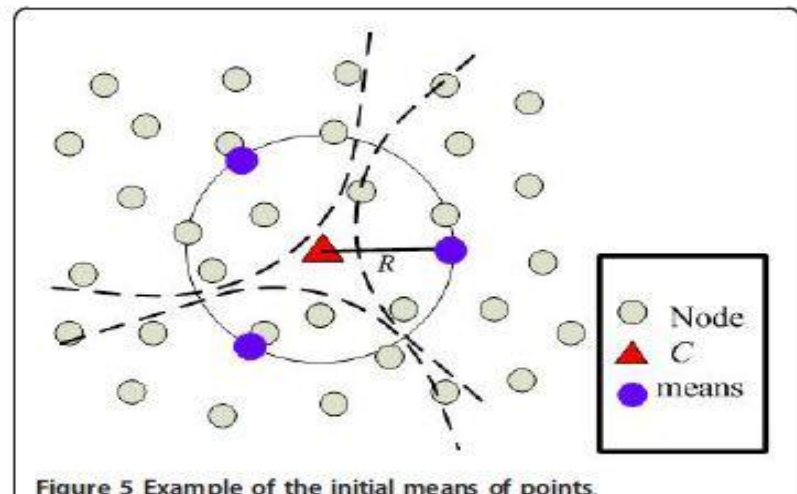

Figure 5 Example of the initial means of points.

We use the k-means algorithm to partition the $\mathrm{n}$ sensor nodes into $\mathrm{k}$ clusters in which each sensor node belongs to the cluster with the nearest mean of point. If there are $\mathrm{k}$ clusters in the system, the k-means function can be expressed by

$$
\operatorname{avg} \min \sum_{i=1}^{k} \sum_{X_{j} \in S_{i}}\left|X_{j}-m_{i}\right|^{2},
$$

where $\mathrm{Si}$ is the cluster $\mathrm{i}, \mathrm{Xj}$ is coordinate of sensor node $\mathrm{j}$ and $\mathrm{mi}$ is the coordinate of mean of point. The main reason for this expression is to obtain the mini- mum average distance between the means of points and the sensor nodes for all clusters.

In order to create uniform distributed clusters, the minimal distance between the means of points and all sensor nodes is calculated. Then, the sensor nodes are classified into the cluster according to the minimal distance. If the $\mathrm{Xj}$ is the closest to the $\mathrm{mi}$ in the th execution, the sensor node $\mathrm{j}$ will join the cluster $i$, which can be expressed by

$$
S_{i}^{(t)}=\left\{X_{j}:\left|X_{j}-m_{i}^{(t)}\right|^{2} \leq\left|X_{j}-m_{i^{*}}^{(t)}\right|^{2} \text { for all } i^{*}=1, \ldots, k\right\},
$$

where each sensor node joins exactly one cluster. The main goal of this expression is to decide which cluster the sensor node $\mathrm{j}$ belongs to in the thexecution.

When the classification of all nodes is done, the new mean of point is created which is calculated by 


$$
m_{i}^{(t+1)}=\frac{1}{\left|S_{i}^{(t)}\right|} \sum_{X_{j} \in S_{i}^{(t)}} X_{j}
$$

where $S(i t)$ is the number of sensor nodes in the cluster $\mathrm{i}$. Figure 6 shows the example of the new means of points.

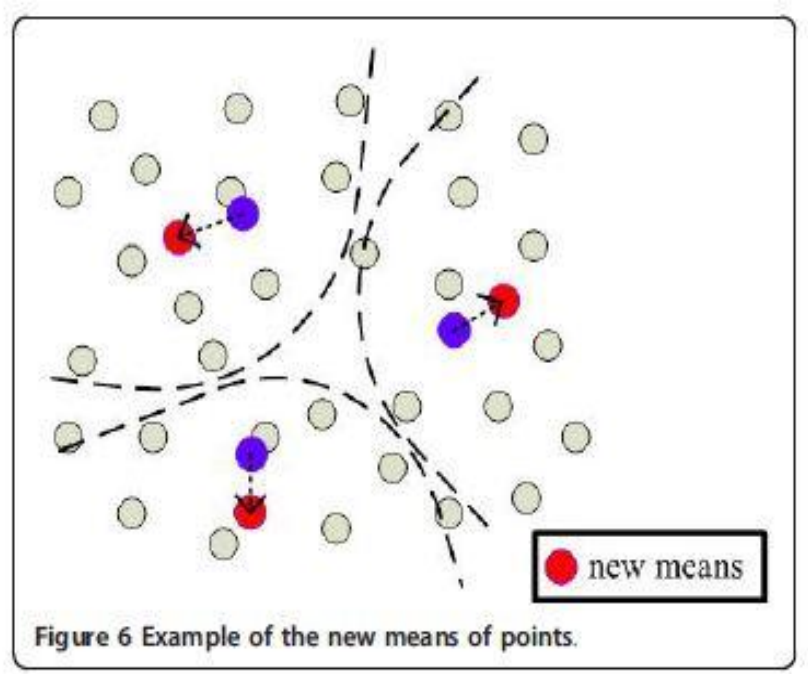

Because the means of points are changed, all sensor nodes are re-classified by executing Equations (9) and (10) iteratively to obtain the minimum average distance between the means of points and the sensor nodes for all clusters. The final clusters are formed when each sensor node is fixed in the cluster. Figure 7 shows the flowchart of the initial cluster processing for our proposed scheme. The cluster head is a sensor node which is closer to the final mean of point and the residual energy of the sensor node is higher than the average residual energy in each cluster. Finally, the cluster architecture is created. The BS broadcasts the routing information of the clusters to all sensor nodes. Hence, each sensor node has its own routing table and knows its task (e.g., cluster head or non-cluster head). Also, each sensor node knows the distances from any other sensor node in its cluster and thereby calculates the transmission power. Based on the number of the sensor nodes within the cluster, the cluster head node creates a schedule based on Time Division Multiple Access (TDMA) to allocate the time for the cluster members.

\subsection{Steady-state phase}

Once the clusters are created and the TDMA schedule is fixed, data transmission can begin. The non-cluster head nodes send data to cluster head node during their allocated transmission time. When all the data have been received, the cluster head node performs signal processing to compress the data into a single signal. Then, this signal is sent to the BS. The amount of information is reduced due to the data aggregation done at the cluster head node. This round is done and the next round begins with set-up and steady-state phases repeatedly

To avoid unnecessary nodes control messages transmission and control overhead of the BS, the clusters are re-created only when the sensor node cannot work in a certain round. So, the calculating overhead is only cluster head selecting in the most set-up phase.

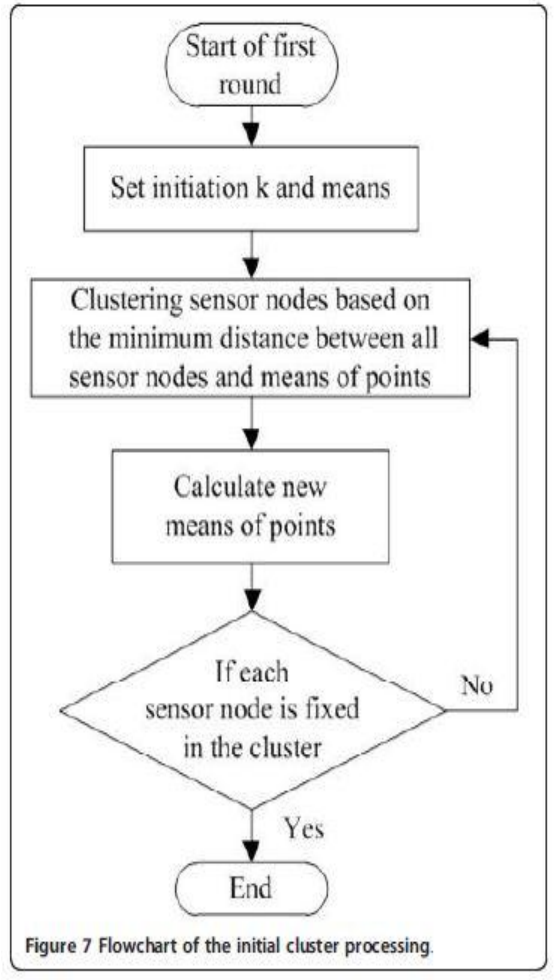

\section{Performance analysis}

In this section, we evaluate the performance of our proposed SECA using a simulation model. We describe our simulation model and illustrate the simulation results, and compare our scheme with the LEACH, HEED, and LEACH-C. We design a simulation environment by using $\mathrm{CH}$. The assumptions for our simulation study are as follows.

- The simulation environment is composed of a BS and some sensor nodes.

- The BS is fixed and located far from the sensor nodes.

- The location of each sensor node is randomly distributed in the sensing area.

- The non-cluster head node can monitor the environment and send data to the cluster head node.

- The cluster head node can gather data, compress it, and forward to the BS.

- All sensor nodes are stationary and the initial energy is the same for each sensor node. All the parameters used in our simulation are listed in Table $1[5,6]$.

\begin{tabular}{ll} 
Table 1 Parameters used in simulation mode \\
\hline Parameter & Value \\
\hline Electronics energy $\left(E_{\text {cicd }}\right)$ & $50 \mathrm{~nJ} / \mathrm{bit}$ \\
Energy for data aggregation $\left(E_{D A}\right)$ & $5 \mathrm{n} / \mathrm{bit} / \mathrm{signal}$ \\
Initial energy of node $\left(E_{\text {int }}\right)$ & $2 \mathrm{~J}$ \\
Packet size & $2000 \mathrm{bits}$ \\
Number of nodes $(n)$ & $50,100,150$ \\
Position of BS $(X, Y)$ & $(50,175)$ \\
Sensing area $(M \times M)$ & $100 \times 100,200 \times 200$ \\
\hline
\end{tabular}

These performance measures obtained on the basis of ten simulation runs are plotted as a function of the rounds and total network energy. A round is defined as the receiving data from all sensor nodes to the BS. The total network energy is defined as the sum of residual energy at all sensor 


\section{International Journal of Science and Research (IJSR) \\ ISSN (Online): 2319-7064 \\ Index Copernicus Value (2013): 6.14 | Impact Factor (2014): 5.611}

nodes. For fair comparison, we set the number of clusters is equal to 5 which is defined in LEACH-C. Figures $\underline{8}$, 9, and 10_show the clustering structure for a certain round in the simulation by using LEACH-C, HEED, and our proposed SECA scheme. According to SECA features, it is intuitive that each cluster size is almost the same and cluster heads locate more closely to the cluster centers. Hence, our proposed algorithm reduces the data transmission distances of sensor nodes and results in the lower energy consumption in the wireless sensor networks.

Figure 11 shows the total network energy when the number of sensor nodes is 50 and the sensing area is $100 \mathrm{~m} \times 100 \mathrm{~m}$. It is evident that the residual energy of our proposed SECA scheme is higher than that of LEACH, HEED, and LEACH$\mathrm{C}$ schemes after 900 runs. This is because our proposed SECA scheme provides the uniform cluster location and better cluster formation. The data transmission distance from each sensor node to its cluster head node is minimized. Thus, the energy consumption is saved.
Figures 12_and 13 show the first sensor node dead and half of the sensor nodes alive for four methods when the number of sensor nodes is 50 and the sensing area is $100 \mathrm{~m} \times 100 \mathrm{~m}$. According to Figures 12and 13, the lifetime of the sensor node of our pro- posed SECA scheme is better than that of LEACH, HEED, and LEACH-C schemes. The reason for this behavior is that the data communications consume a large amount of energy in wireless sensor networks. However, the transmission distance between non-cluster head node and cluster head node is suitable by using our scheme. The transmission power of non- cluster head nodes are reduced. Also, in order to prove our proposed scheme is well designed, we increase the number of sensor nodes in the simulation environment. Figure 14_shows the total network energy when the number of sensor nodes is 100 and the sensing area is $100 \mathrm{~m} \times 100 \mathrm{~m}$. Due to the better energy saving approach in the proposed scheme, according to SECA features, it is intuitive that the proposed scheme results in higher residual energy than LEACH, HEED, and LEACH-C schemes when the sensor node increases in the same sensing area.
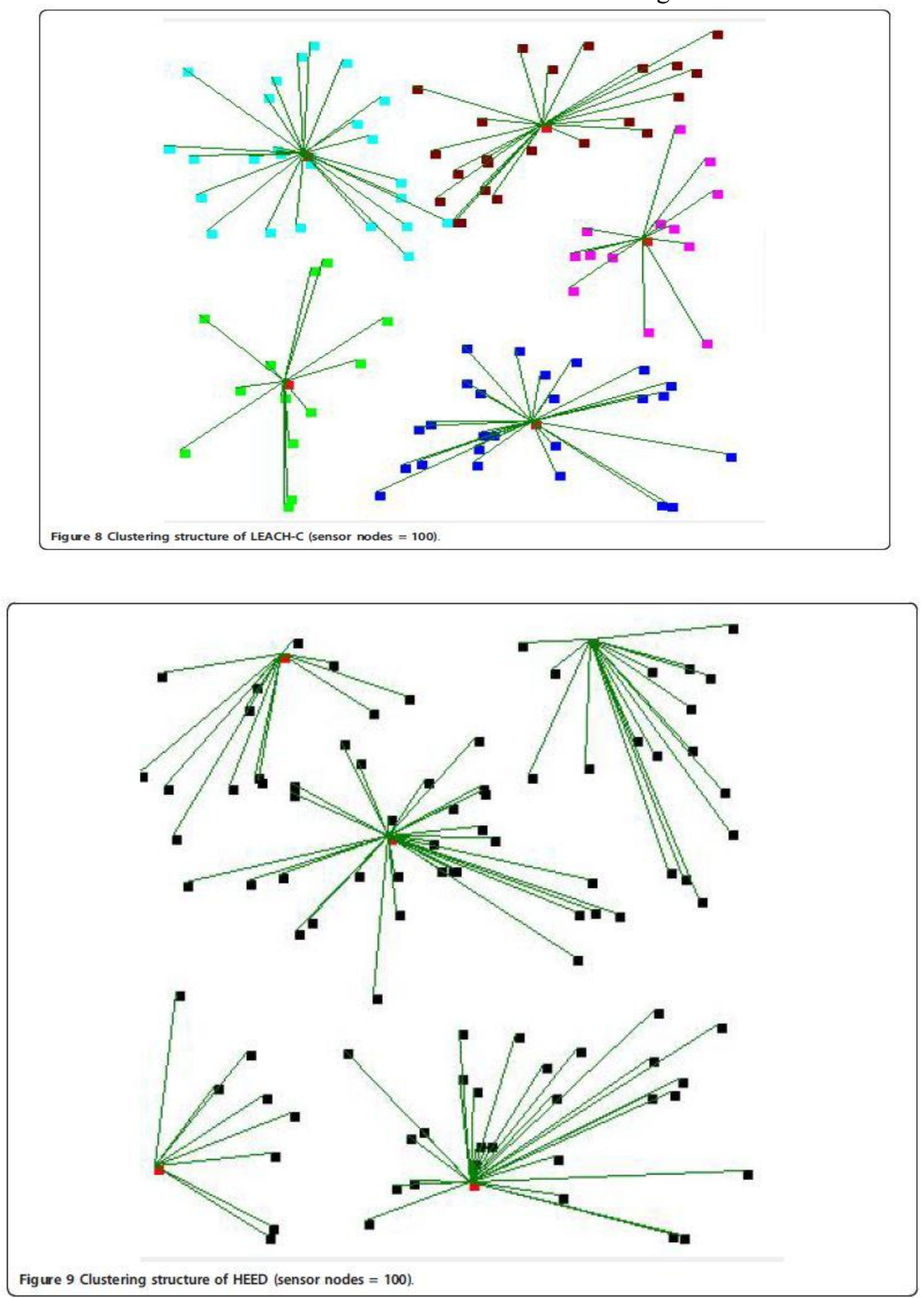

Volume 4 Issue 11, November 2015 


\section{International Journal of Science and Research (IJSR) \\ ISSN (Online): 2319-7064}

Index Copernicus Value (2013): 6.14 | Impact Factor (2014): 5.611

Furthermore, we extend the sensing area to $200 \mathrm{~m} \times 200 \mathrm{~m}$ in the simulation environment. Figure 15 shows the total network energy when the number of sensor nodes is 150 . The distributions of clusters in LEACH and HEED are not uniform and some clusters consist of huge number of sensor node in a large area. Hence, the cluster head of the cluster with huge number of sensor nodes will suffer from heavy traffic load and result in significant energy consumption. According to Figure 15, the curves indicate that our scheme improves the energy utility more significantly than LEACH, HEED, and LEACH-C schemes when the sensing area is extended. From the simulation results, it is clear that our proposed scheme strikes the appropriate performance in the energy consumption and network lifetime for the wireless sensor networks.

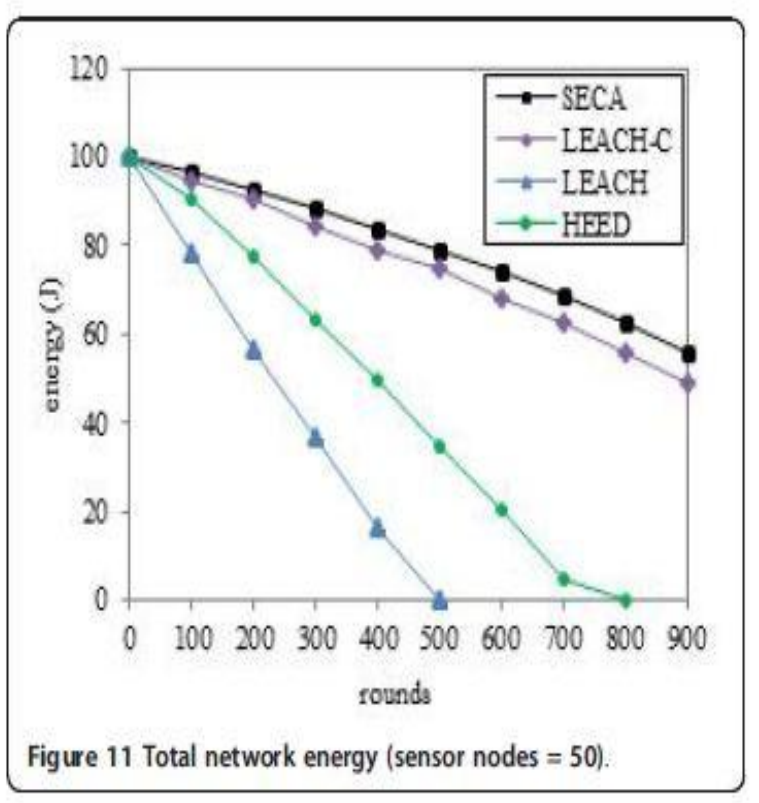

\section{Conclusions}

The energy saving is a challenging issue in the wireless sensor networks. To increase energy efficiency and extend the lifetime of sensor node, new and efficient energy saving schemes must be developed. In the pro- posed scheme, we calculate the average distance between the sensor nodes and take into account the residual energy for selecting the appropriate cluster head nodes. The lifetime of wireless sensor networks is extended by using the uniform cluster location and balancing the network loading among the clusters. Simulation results indicate our proposed algorithm achieves the low energy consumption and better network lifetime in the wireless sensor networks
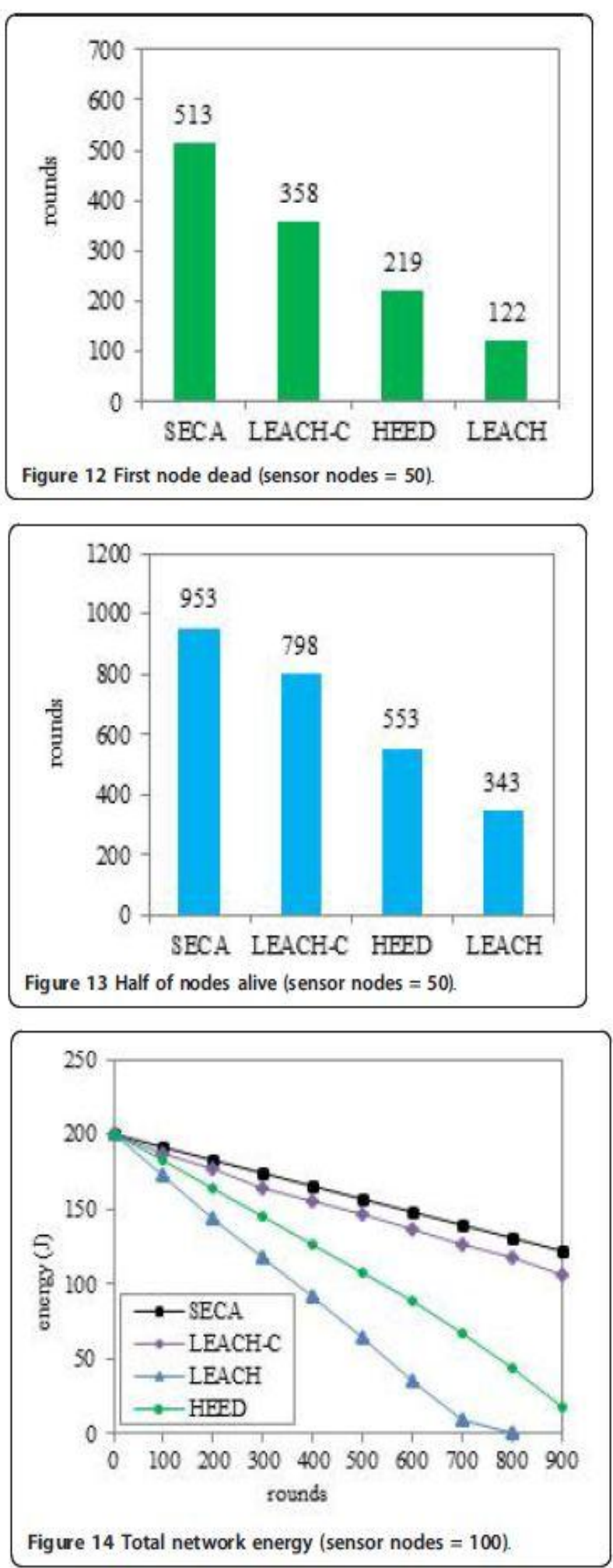


\section{International Journal of Science and Research (IJSR) \\ ISSN (Online): 2319-7064}

Index Copernicus Value (2013): 6.14 | Impact Factor (2014): 5.611

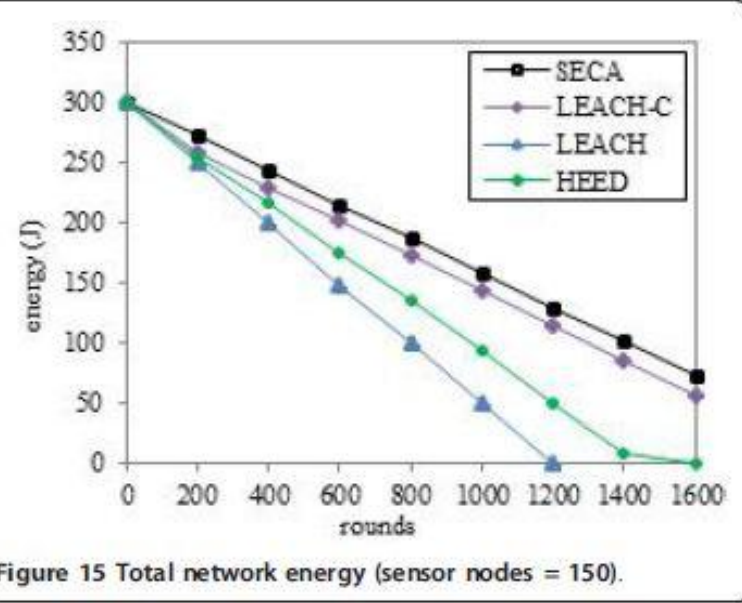

\section{References}

[1] IF Akyildiz, W Su, Y Sankarasubramaniam, E Cayirci, A survey on sensor networks. IEEE Commun Mag. 40(8), 102-114 (2002). doi:10.1109/ MCOM.2002.1024422

[2] M Tubaishat, S Madria, Sensor networks: an overview. IEEE Potentials. 22(2), 20-23 (2003)

[3] JN Al-Karaki, AE Kamal, Routing techniques in wireless sensor networks: a survey. IEEE Wirel Commun. 11(6), 6-28 (2004). doi:10.1109/ MWC.2004.1368893

[4] A Chamam, S Pierre, On the planning of wireless sensor networks: energy efficient

[5] Clustering under the joint routing and coverage constraint. IEEE Trans Mob Comput. 8(8), 1078-1086 (2009)

[6] WR Heinzelman, A Chandrakasan, H Balakrishnan, Energy-efficient communication protocol for wireless micro sensor networks, in Proc $33^{\text {rd }}$ Hawaii International Conference on System Sciences, pp. 1-10 (January 2000)

[7] WB Heinzelman, P Chandrakasan, H Balakrishnan, An application-specific protocol architecture for wireless micro sensor networks. IEEE Trans Wirel Commun. 1(4), 660-670 (2002). doi:10.1109/TWC.2002.804190

[8] O Younis, S Fahmy, HEED: a hybrid, energy-efficient, distributed clustering approach for ad hoc sensor networks. IEEE Trans Mob Comput. 3(4), 366379 (2004).

[9] S Babaie, AK Zadeh, MG Amiri, The new clustering algorithm with cluster members bounds for energy dissipation avoidance in wireless sensor network, in Proc Computer Design and Applications (ICCDA), pp. 613-617

[10](June 2010)

[11]Q Xuegong, C Yan, A control algorithm based on double cluster-head for heterogeneous wireless sensor network, in Proc Industrial and Information Systems (IIS), pp. 541-544 (July 2010)

[12] Y-U Yun, J-K Choi, N Hao, S-J Yoo, Location-based spiral clustering for transmission scheduling in wireless sensor networks, in Proc Advanced Communication Technology (ICACT), pp. 470-475 (February 2010)

[13]HD Tarigh, M Sabaei, A new clustering method to prolong the lifetime of WSN, in Proc Computer
Research and Development (ICCRD), pp. 143-148 (March 2011)

[14] T Kanungo, DM Mount, NS Netanyahu, CD Piatko, R Silverman, AY Wu, An efficient k-means clustering algorithm: analysis and implementation. IEEE Trans Pattern Anal Mach Intell. 24(7), 881-892 (2002). doi:10.1109/

[15] TPAMI.2002.1017616

[16]J Zhu, H Wang, An improved K-means clustering algorithm, in Proc 2nd IEEE International Conference on Information Management and Engineering(ICIME), pp. 190-192(2010) 